\title{
Bevel Gearbox Fault Diagnosis using Vibration Measurements
}

\author{
Dennis Hartono ${ }^{1}$, Dunant Halim ${ }^{1}$, Achmad Widodo ${ }^{2}$ and GethinWyn Roberts ${ }^{3}$ \\ ${ }^{1}$ Department of Mechanical, Materials and Manufacturing Engineering, The University of Nottingham Ningbo China, China 314100 \\ ${ }^{2}$ Department of Mechanical Engineering, Diponegoro University, Semarang, Indonesia 50275 \\ ${ }^{3}$ Department of Civil Engineering, The University of Nottingham Ningbo China, China 315100
}

\begin{abstract}
The use of vibration measurementanalysis has been proven to be effective for gearbox fault diagnosis. However, the complexity of vibration signals observed from a gearbox makes it difficult to accurately detectfaults in the gearbox. This work is based on a comparative studyof several time-frequency signal processing methods that can be used to extract information from transient vibration signals containing useful diagnostic information. Experiments were performed on a bevel gearbox test rig using vibration measurements obtained from accelerometers. Initially, thediscrete wavelet transform was implementedfor vibration signal analysis to extract the frequency content of signal from the relevant frequency region. Several time-frequency signal processing methods werethen incorporated to extract the fault features of vibration signals and their diagnostic performances were compared. It was shown thatthe Short Time Fourier Transform (STFT) could not offer a good time resolution to detect the periodicity of the faulty gear tooth due the difficulty in choosing an appropriate window length to capture the impulse signal. The Continuous Wavelet Transform (CWT), on the other hand, was suitable to detection of vibration transients generated by localized fault from a gearbox due to its multi-scale property. However, both methods still require a thorough visual inspection. In contrast, it was shown from the experiments that the diagnostic method using the Cepstrumanalysis could provide a direct indication of the faulty tooth without the need of a thorough visual inspection as required by CWT and STFT.
\end{abstract}

\section{Introduction}

In order to accurately diagnose possible faults in a gearbox, theextraction of features from the impulsive signal associated with the gearbox vibration is crucial. Alarge number ofsignal processing methodshave beenproposedto identify the impulsive features from vibration signals, particularly using time-frequency analysis such as the Wigner-Ville distribution,Cepstrum,Fast Fourier Transform (FFT), and the amplitude-phase demodulation.

In most cases, vibration signals from a faulty gearbox will consist of a series of impulses that repeat at a particular defect frequency [1].An impulsesignal is a nonstationary signal that cannot be captured by conventional steady-state spectrum analysis, such as FFT analysis, because of the main assumption of FFT analysis that the underlying signal must be stationary.

The Short Time Fourier Transform (STFT) [2-4], however, can overcome the aforementioned problem. However, the main problem with the method concerns about the constant resolution for all frequencies because it uses the same window for the analysis of the entire signal. On the other hand, the CWT has a multi-scale property alower scale wavelet hasa high time resolution and a lower frequency resolution, but a higher scale wavelet has a high frequency resolution and a low time resolution[5]. Compared to STFT, CWT has the flexibility in adjusting its time-frequency resolutionwhich means thatit is more suitable to analyzethe impulsive signals associated with a faulty gearbox.

On the other hand, Cepstrum analysis has been widely used for signal periodicity detection in the frequency spectrum, especially for speech analysis that contains harmonics in its voice frequencybandwidth.Cepstrum can be considered as the frequency spectrum of a frequency spectrum.In gearbox vibration signals, harmonics in the form of sidebands equally spaced at an integer multiple of carrier frequencies or gearmesh frequencies. The defects that introduced as the periodic patterns in the vibration signals can be considered as a system with multiple echoes [6]. Therefore,Cepstrum can be used to identify the periodicity observed from the harmonics associated with certain gear faults.

The objective of this study is to investigate theeffectiveness of severaltime, frequency or joint timefrequency signal processing methods to identify the fault location in a gearbox. The need of each method for a thorough visual inspection for the fault location identification was investigated. Once the vibration measurements were obtained from the gearbox, FFT was carried out to evaluate the frequency contents of the signal.Discrete Wavelet Transformwas thenused to extract the useful frequency bandwidth to be analysed further.Finally, the performance ofseveral signal 
processing techniques were compared for gearbox fault diagnosis.

\section{Theoretical Background}

\subsection{Discrete Wavelet Transform}

The Discrete Wavelet Transform (DWT) is an alternative method of the Continuous Wavelet Transform, which is discretized in terms of scale and translations. It is widely used in signal compression and filtering [7, 8]. Equations 1-4 providethe basic analysis of DWT decomposition scheme

$$
\begin{aligned}
& \mathrm{x}(t)=\hat{x}_{0}(t)+\sum_{n=-\infty}^{\infty} \sum_{m=-\infty}^{0} T[m, n] \Psi_{m, n}(t)(1) \\
& \hat{x}_{0}(t)=\sum_{n=-\infty}^{\infty} S[0, n] \emptyset(t-n) \\
& \Psi_{m, n}(t)=a_{0}{ }^{-\frac{m}{2}} \psi\left(\mathrm{a}_{0}{ }^{-m} t-n b_{0}\right)(3) \\
& \emptyset_{m, n}(t)=1 / 2^{\frac{m}{2}} \emptyset\left(2^{-m} \mathrm{t}-\mathrm{n}\right)
\end{aligned}
$$

(4)where $\psi_{m, n}(t)$ and $\emptyset_{m, n}(t)$ are the respective scaled wavelet and scaling functions, $a_{0}=2$ and $b_{0}=1 . T[m, n]$ is a typical wavelet coefficient. $S[0, n]$ is the aggregate of all details for scales greater than or equal to 1 (coarser scale). In practice, DWT is normally implemented using a series of filter banks. Interested reader can refer to [9] for further details.

\subsection{Short Time Fourier Transform (STFT)}

The STFT is considered as the most straight forward time-frequency signal processing methods. It is based on slicing the waveform of interest into several segments and then performing the usual FFT analysis on each of these segments. The usual FFT is applied within each segment. The idea is that the underlying signal is assumed to be stationary within each segment.It is formulated as [10]:

$\mathrm{X}(t, f)=\int_{-\infty}^{\infty} \mathrm{x}(\tau) \mathrm{w}(t-\tau) \mathrm{e}^{-j \pi f \tau} d \tau$

where $\mathrm{w}(t-\tau)$ is the window function and $\tau$ is the variable that slides the window across the underlying waveform.

\subsection{Continuous Wavelet Transform (CWT)}

The Continuous Wavelet transform is a linear transformation decomposing the underlying signal $\mathrm{x}(t)$ into elementary functions called wavelets. Wavelets are local functions in time that can be described as[11]:

$W(a, b)=\frac{1}{\sqrt{a}} \int_{-\infty}^{\infty} \mathrm{x}(t) h^{*}\left(\frac{t-b}{a}\right) d t(6)$ where the basis function for the wavelettransformisgiven in term of translation parameterbanddilation parameter $a$ with the followingmotherwaveletfunction:

$$
h_{a, b}(t)=\frac{1}{\sqrt{a}} h^{*}\left(\frac{t-b}{a}\right)
$$

\subsection{Cepstrum}

Cepstrum can be regarded as the spectrum of a spectrum. It can detect the periodicities in the frequency domain. There are several forms of Cepstrum analysis, yet most of them can be considered as a spectrum of a logarithmic spectrum as formulated below[12]:

$$
C_{p}(\tau)=F^{-1}\left(\log \left[S_{x x}(f)\right]\right)
$$

where $\tau$ isquefrency (s), $S_{x x}$ isusual FFTspectrum and $F^{-1}$ is the Inverse Fourier Transformmethod.

\section{Experimental Apparatus}

Experimentson asingle-stage bevel gearbox were carried out on atest-rig from the Machinery Fault SimulatorSpectra Quest located at Diponegoro University as shown in Figure 1.

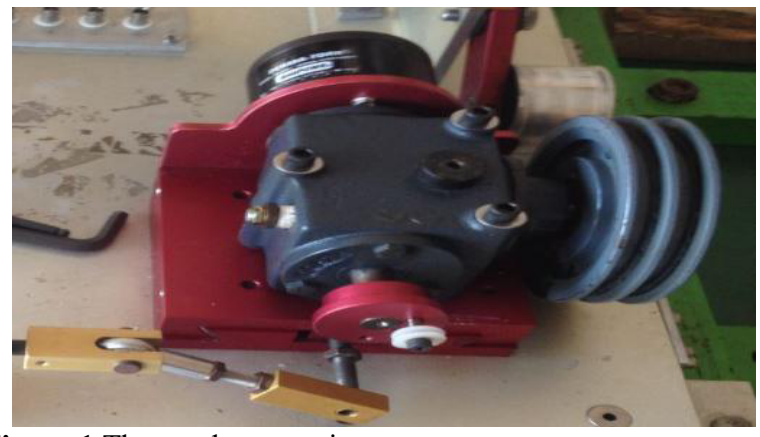

Figure 1.The gearbox test rig.

During the experiment, a three-phase AC motor was used as the driver of the system and amagnetic brake with the third scale was incorporated as a load. Three accelerometers sensor were used to capture the vibration signals from the test rig,although only the axial measurementwas used in the analysis. Tooth breakage gear fault was introduced in this work and compared with the healthy gear. The broken gear usedin the experiment was depicted at Figure 2.

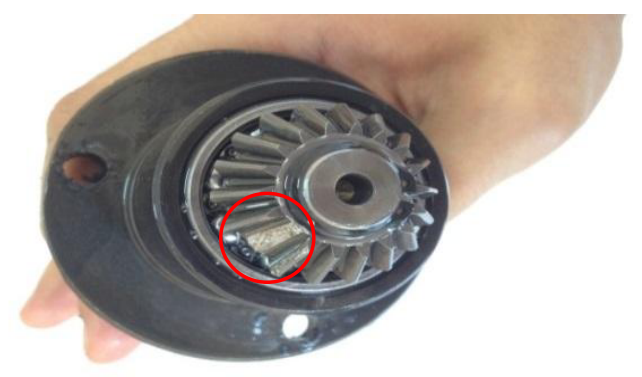

Figure 2. The bevelgear with a faulty tooth. 
For the experiment, $5.12 \mathrm{kHz}$ sampling rate was used to sample the vibration signals to gather eight seconds of data. A tachometer sensor from Lattice Instruments was also used to provide the phase information from the gear, which then will be used to pre-process the vibrations signal by TSA method to get rid of the asynchronous component from the faulty gear under consideration.

\section{Results and Discussions}

The raw vibration signal (in terms of axial acceleration output from the accelerometer) of a faulty gear in axial direction is presented in Figure 3. It can be seen that it is not straightforward to extract the fault information by merely observing the time domain behaviour of the raw vibration signal. There are several observed spikes that occurred and may provide useful information for detecting the periodicity of impulsive signal from the fault gear. However, the spikes may also be contributed by the measurement noise obtained during the data acquisition process.

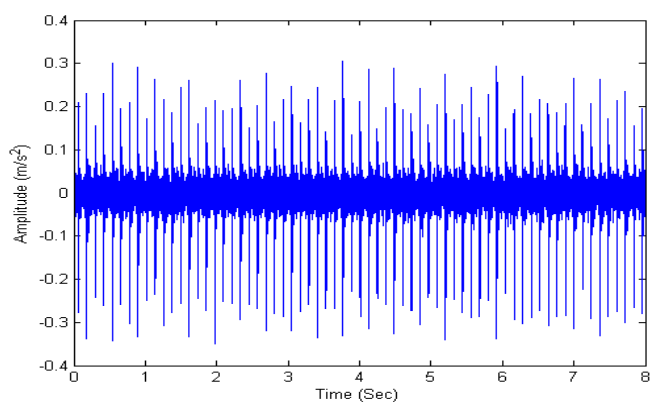

Figure 3.Vibration signal from a faulty gearbox.

Another method to analyze the behaviour of the signal from the faulty gear is by utilizing the spectral analysis of FFT as presented in Figure 4. As mentioned in introduction, gearbox signals are characterized bysidebands around gearmesh frequency and its harmonics. Sidebands can thus provide the useful information that indicates the faulty tooth existence [13].

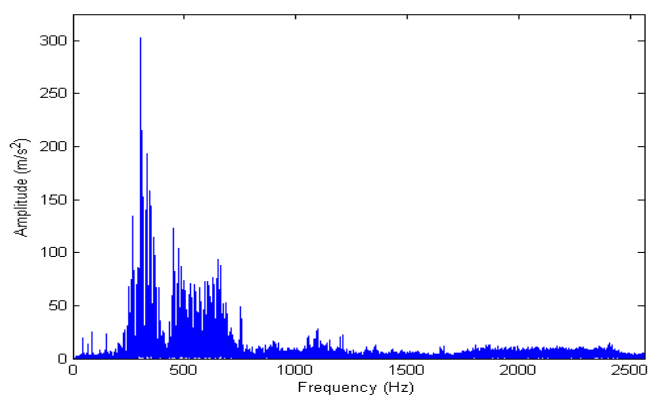

Figure4.FFT of vibration signal from a faulty gearbox.

FFT can show sidebands patterns,offering diagnostic information that is harder to visualize from time domain. However, it does not contain information about how frequency contents evolve over time and it is not suitable to analysenon-stationary signal, such as impulse signal typically observed in this situation. Moreover, it is usually impossible to observe the sidebands around gearmesh harmonics becausesomesidebandscanbecovered by the noisethatcan havehigher amplitudes compared to those of the sidebands.

For further analysis, DWT is utilized to extract useful information from the signal from the faulty gear. From FFT plot in Figure 4, it can be concludedthat the important frequency bandwidth that needs to be analyzedis approximately in the range of $0-700 \mathrm{~Hz}$ due to strong amplitude of sidebands around third and fourth gearmesh frequencies.This can be achieved by decomposing the vibration signals by 3 level DWT so it can provides signals in frequency range from 0-640 Hz. Due to the fact that the experiment was carried out under steady-state condition, only $0.4 \mathrm{~s}$ segmented part of the de-noised axial vibration signal was chosen to be furtheranalyzed by the time-frequency analysis.

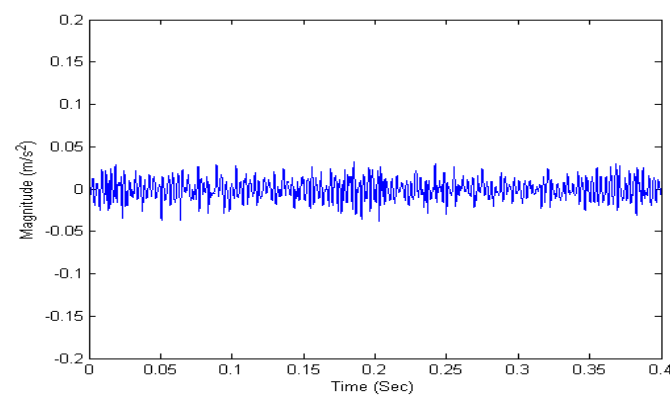

Figure 5.DWT of vibration signal from a healthy gearbox.

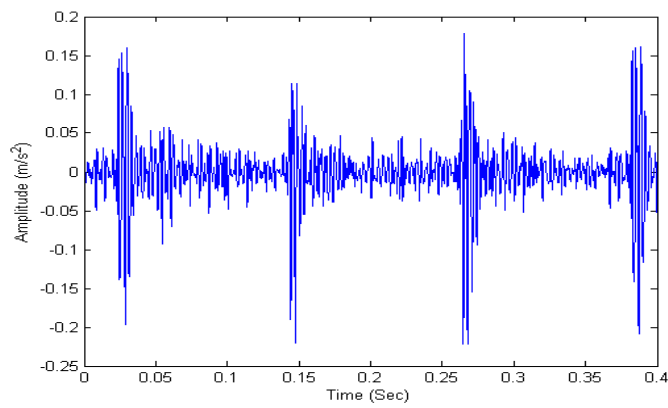

Figure 6.DWT of vibration signal from a faulty gearbox.

Figures5 and 6show the signals after DWT decomposition for healthy and faulty gearboxes, respectively. It is observed that the healthy gearbox shows a regular pattern of vibration amplitude compared to the broken gearbox. The plot also showsthe impulsive patterns that correspond to the impact generated by faulty tooth when it meshed with the other gear. Another way to extract the periodicity of signal is by analysing the time domain signal in the joint time-frequency representation. Such a representation isnot only able to provide the time representation of the signals but also able to provide information about how the frequency contents of the underlying signal evolve over time, which cannot be achieved by FFT analysis. To obtain such a representation, another method for the time-frequency analysis will be needed as presented in this work. 


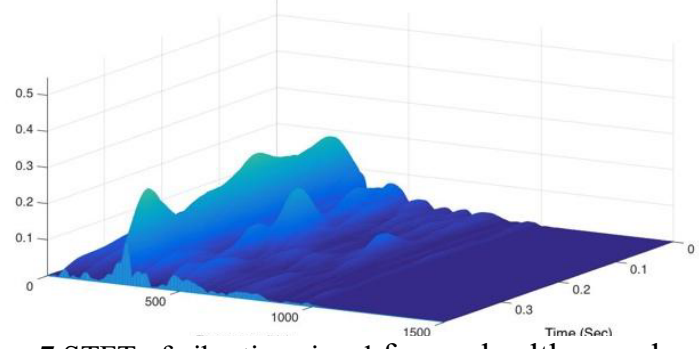

Figure 7.STFT of vibration signal from a healthy gearbox.

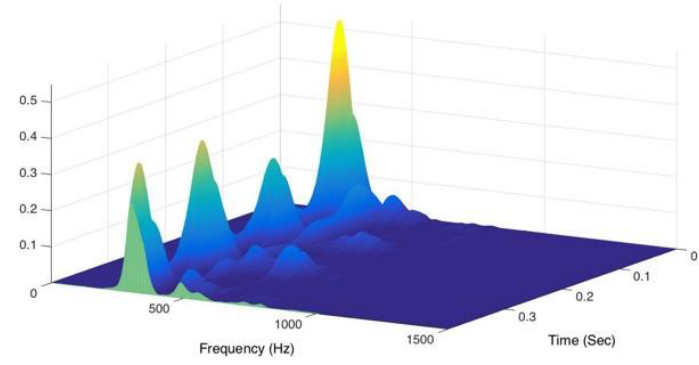

Figure 8.STFT ofvibration signalfrom a faulty gearbox.

Initially,STFT was incorporated to capture the impulse signal characteristics from the vibration signal. STFT is considered as the simplest method for timefrequency analysis to evaluate how the frequency components of signals evolve over time. STFT plot of healthy and broken gearboxes are presented in Figures7 and 8, respectively. As observed from the figures,signalsassociated with healthy and broken gearboxes can be differentiated based on their patterns. However, the constant time-frequency window used in the analysis usually makes STFTnot suitablefor analyzing complex vibrations signals that require the multi scale signal analysis[5]. In other words, once the length of the window is chosen, the entire analysis will be based onfixed time and frequency bandwidths. Figure 8 showed clearly that the poor time resolution of STFT in presenting the precise timing of the impulsive event. This timing is quite important to determine the time period of the faulty gear that can be used to identify which gear contains the fault[7].

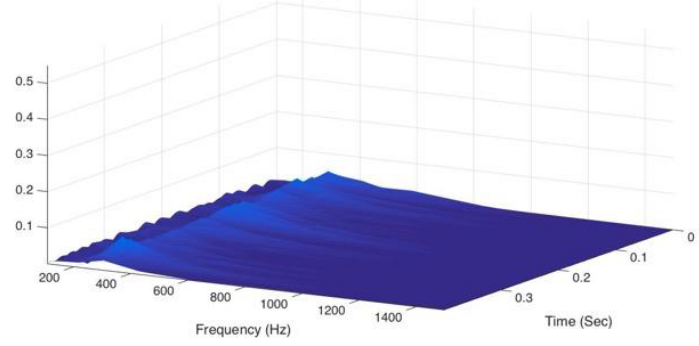

Figure 9.CWTof vibration signalfrom a healthy gearbox.

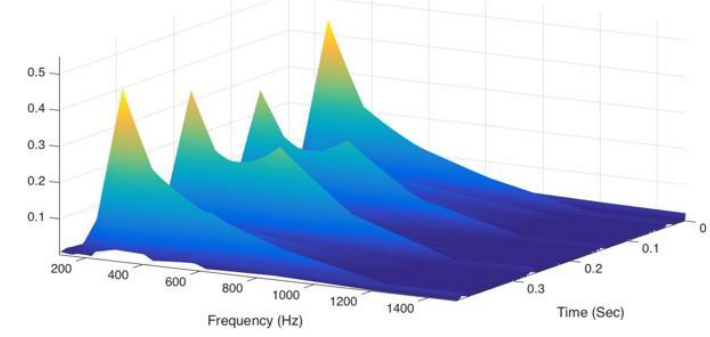

Figure 10.CWTof vibration signal from a faulty gearbox.

The CWT as one of the most prominent tools in detecting transient signals offers a better compromise for time-frequency signal analysis. It has a fine localization of frequency content in the low frequency region and a fine localization of time contentat the high frequency region, which makes it as an excellent tool to diagnose gearbox fault[5]. As the impulsive feature of vibration signal exhibits a short time duration but a wide frequency bandwidth, CWT is one of the best candidates to be used to detect the pattern of vibration signal from the faulty gear. Figures9 and 10represented the scalogram (the magnitude of CWT coefficients) plots of vibration signal from the healthy and faulty gearboxes.Figure 10 clearly revealed the transient signal characteristics and the period of the impulse signal that reflected the rotation frequency of the faulty toothusing the joint time-frequency representation. The signal periodwas approximately 0.1195 second between two successive impulses. These two figures showed that CWT was not only able to distinguish the pattern between the healthy and broken gears but also offered a better time resolution to detect the periodicity between two impulses, compared to STFT.Since the periodicity was inversely related to the speed of the faulty gear, so the method was also able to determine which gear was generating the impulse due to faulty tooth.

Although CWT overcamethe STFT limitations,it still requireda thorough inspection to the plot of the scalogramto provide information about thefaulty gears, which was not a straight forward process. Based on this consideration, the Cepstrum method was investigated since it could offer a better solution to detect the signal from the faulty gears. This can be doneby detecting the sidebands pattern from the signal from the faulty gears around the gearmesh frequency that presents in FFT, sincecepstrum reveals the periodicity of spectrum [12]. Since gearbox vibration typically contains harmonics in itsspectrum(e.ggearmesh frequency, sidebands) which makes Cepstrum a good candidate to detect such harmonics for gear fault diagnosis. Figure 11 showed the Cepstrum of vibration signal from the faulty gearbox. The quefrency of the signal from the faulty gear was observed to be $0.11947 \mathrm{~s}$.This indicated that there were some sidebands around the gearmesh frequency spaced at 8.37 $\mathrm{Hz}$ (i.e. 1/0.11947), which corresponded to the rotational frequency of the faulty gear.It was shown that Cepstrum method could eliminate the need for a manual visual inspection for determining the periodic pattern of the faulty tooth signal, in contrast to the CWT method. 


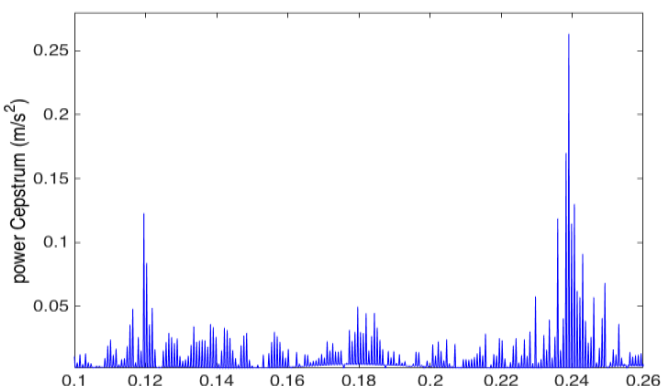

Figure 11.Cepstrum of vibration signal from a faulty gearbox.

\section{Conclusions}

A comparative studyof several gear fault diagnostic methods using vibration measurements has been done. The aim of the study is to detect the existence of a faulty gear with a broken tooth from a bevel gearbox testrig.Several time-frequency signal processing methods were compared: FFT, STFT, CWT and Cepstrum methods.Initially, DWT was implemented to extract only the useful frequency content of the raw vibration signal that containedthe fault features. It was further observed thatFFT could reveal the sideband patterns that contained information about which particular gear contained a faulty tooth. However, such informationwas difficult to identify due to the need for thorough visual inspections of such sidebands that might be corrupted by the measurement noise. In this case, STFT offereda better solution by providing the simultaneous time-frequency analysis to detect the impulse signals generated by the faulty tooth. Itsusefulness for fault diagnosis, however,was still restricted by theduration-bandwidth principle that compromisedthe time and frequency resolutions. Appropriate window function and length should thus be taken care to be able to extract the gear fault features. In this work, STFT gavea poor time resolution, which is insufficient to indicate the accurate timing of fault features generated by gearbox. On the other hand, CWT has been shown to be a better candidate to analyze thetransient signals since it was able to offer a better solution compared to STFT. However,a thorough visual inspection was still required. Finally, the experiments showed the advantage of the Cepstrum analysis in providing the gear fault information without the need of a thorough visual inspection.

\section{Acknowledgment}

The authors gratefully acknowledge the support provided by Ningbo Science and Technology Bureau-International Cooperation Programme(Project: 2012D10029), China.

\section{References}

1. R.B. Randall,Vibration-based Condition Monitoring: Industrial, Automotive, and Aerospace Applications (2011)

2. G. Chen, J. Chen, G. Dong, H. Jang, Appl. Acst87, 131-141 (2015)
3. G. He, K. Ding, W. Li. X. Jiao, Ren. Engy87, 364375 (2016)

4. O. D. Mohammed, M. Rantatalo, Mech. Sys. Sig. Proc66-67. 612-624 (2016)

5. L. Jedlinski, J. Jonak, Appl. Sft. Cmpt30, 636-641 (2015)

6. S. Jolivet, S. Mezghani, M. E. Mansori, B. Jourdain, J. Mnfct. Sys 37, 467-471 (2015)

7. S. Jin, J. S. Kim, S. K. Lee, J. Mech. Sci. Tech 29, 3165-3173 (2015)

8. V. Skrickij, M. Bogdevicius, R. Juvenicius, Appl. Acst106. 51-62 (2016)

9. S. Mallat, A Wavelet Tour of Signal Processing, The SparseWay(2009)

10. N. H. Chandra, A. S. Sekhar, Mech. Sys. Sig. Proc72-73, 105-133 (2016)

11. W. He, Q. Miao, M. Azarian, M. Pecht, Mech. Sys. Sig. Proc64-65, 149-161 (2015)

12. B. Liang, S.D. Iwnicki, Y. Zhao, Mech. Sys. Sig. Proc 39, 342-360 (2013)

13. G. Dalpiaz, A. Rivola, R. Rubini, Mech. Sys. Sig. Proc14, 387-412 (2000) 